\title{
Zika Clinic: A Health Systems Model for Managing Emerging Infectious Diseases
}

\author{
Aileen Y. Chang ${ }^{1 *}$, Jane Wu ${ }^{1}$, Priyanka Kamalapathy ${ }^{1}$, Homa Ahmadzia ${ }^{2}$, Nicole Swanson ${ }^{1}$, Jeffrey M. Bethony ${ }^{3}$, Gary L. Simon ${ }^{1}$, \\ April Barbour ${ }^{1}$
}

${ }^{1}$ Department of Medicine, The George Washington University, Washington, USA

${ }^{2}$ Department of Obstetrics and Gynecology, The George Washington University, Washington, USA

${ }^{3}$ Department of Microbiology, Immunology, and Tropical Medicine, The George Washington University, Washington, USA

"Corresponding author: Aileen Y. Chang, Department of Medicine, The George Washington University, United States, 2150 Pennsylvania Ave Suite 5-416 Washington, USA. Tel:+12027412222; +19178341181; Fax:+12027412185; Email: chang@gwu.email.edu

Citation: Chang AY, Wu J, Kamalapathy P, Ahmadzia H, Swanson N, et al. (2018) Zika Clinic: A Health Systems Model for Managing Emerging Infectious Diseases. J Hosp Health Care Admin: JHHA-101. DOI: 10.29011/JHHA-101.00001

Received Date: 14 August, 2017; Accepted Date: 09 January, 2018; Published Date: 18 January, 2018

\begin{abstract}
Objective: In response to the recent Zika epidemic, the George Washington University Medical Faculty Associates established a Zika Clinic.

Methods: Zika Clinic provided evidence based Zika virus testing and counseling for reproductive concerns amidst the evolving epidemic and changing testing recommendations.

Results: From October 2016 to March 2017, seventy patients were seen in Zika Clinic all of whom underwent Zika diagnostic testing. The patients were predominantly women $(45 / 70,64 \%)$ and of those $73 \%$ were pregnant. The Zika Clinic sessions were scaled up to meet the needs of increasing patient demand.

Conclusion: This model for the development of an outpatient clinic for an emerging infectious disease proved to be an efficient and scalable method for a healthcare system to address patient care needs during an evolving epidemic with up to date evidence based recommendations and compliance with changing department of health recommendations.
\end{abstract}

Keywords: Clinic; Emerging Infectious Disease; Health Systems; Prenatal Counseling; Zika; Zika Diagnostic Testing

\section{Introduction}

Zika virus is a flavivirus spread by mosquitos. In February 2016, the World Health Organization declared the development of an epidemic in the Americas a public health emergency of international concern [1]. As the Zika cases mounted, an increasing number of patients sought advice from their physicians $[2,3]$. Unfortunately, there was little reliable data regarding sexual transmission, transmission via blood transfusion, the risk of microcephaly, and the likelihood of Guillain-Barré syndrome [4]. Moreover, there was a lack of accurate diagnostic tests due to cross reactivity with dengue virus [4] and the clinical criteria for authorizing Zika diagnostic testing was constantly changing
[5]. Recommendations from the Center for Disease Control and local Departments of Health were in constant flux as the epidemic emerged and new scientific developments arose [5].

This environment posed a challenge for health care providers to provide reliable, evidence based counseling and testing in order to manage the influx of concerned patients. In October 2016, the Division of General Medicine in the George Washington University Medical Faculty Associates (GWUMFA) established the Zika Clinic to provide a site for diagnostic testing and counseling for patients who may have been exposed to this virus. In the face of rapidly changing recommendations from both the Centers for Disease Control and the local Department of Health this model proved to be an efficient and scalable method for a large healthcare system to address patient care needs during an evolving epidemic. 


\section{Materials and Methods}

\section{Zika Clinic Operation}

Two nurse practitioners and two physician assistants in the Division of General Internal Medicine manage the Zika Clinic at the GWUMFA. The clinic was established to provide travelers (and those who might be sexually exposed to travelers) with Zikarelated consultations and arrange appropriate testing through Department of Health of the District of Columbia (DC DOH) or through a commercial laboratory. Initially there was one clinic session per week, but it was expanded to two sessions per week in March 2017 to accommodate rising demands. Figure 1 shows the attendance to Zika clinic per week. Patients were referred from providers from the Departments of Obstetrics and Gynecology and Medicine, the MFA infertility center as well as other institutions in the Washington DC metropolitan area.

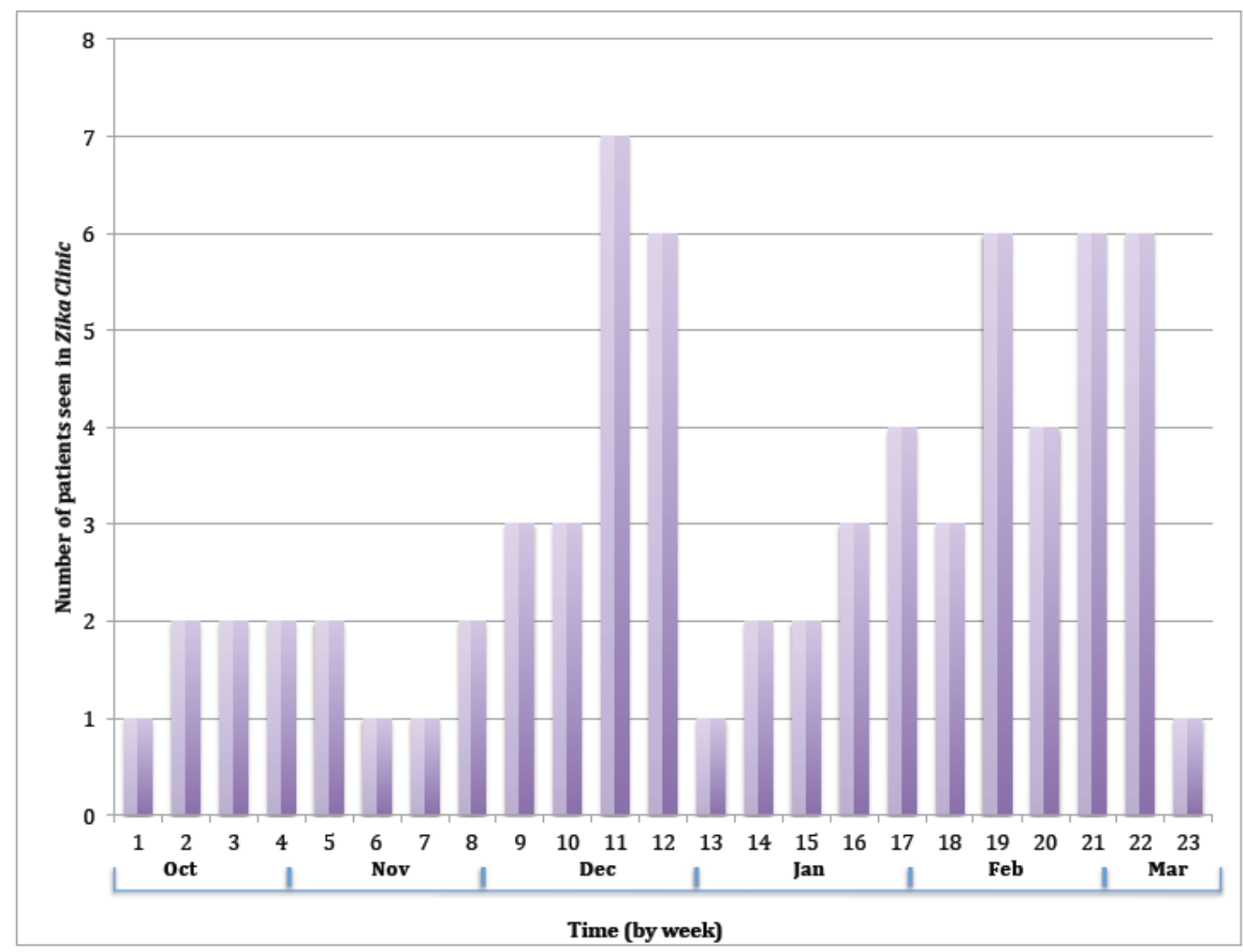

Figure 1: Zika Clinic patient attendance by week.

Ongoing training for Zika Clinic healthcare providers was based on a multi-faceted approach. Providers kept up to date with the Center for Disease Control (CDC) website and research articles, attended meetings held by the DC DOH/ Public Health Laboratory (PHL) on updated testing protocols, engaged in ongoing regular meetings with supervising physicians specializing in Zika research, and met regularly with Zika Clinic providers to share information as it evolved. Providers also joined a listserv for Health Notices from the DC DOH, which provided updates on local guidelines on Zika virus disease management and testing.

There were two testing systems available. DC DOH offered free Zika virus serum and urine testing using Polymerase Chain Reaction (PCR) and Zika IgM antibody testing for people who met defined criteria. As time went on, these criteria expanded as the recognized spectrum of infection increased. LabCorp, a commercial laboratory, also offers a comprehensive Zika test which is a nonFDA approved PCR for use in serum and urine for USD \$780 and MAC-ELISA IgM test for USD \$150. PCR tests are recommended within two weeks of exposure and MAC-ELISA IgM is recommended between 2-12 weeks after Zika virus exposure. All test results received from $\mathrm{CDC} / \mathrm{DOH} / \mathrm{PHL}$ and LabCorp are scanned into patients' electronic medical record. We have a system to record those patients' test sources, type of tests, pending results, repeated results and final status. Patients are notified of their test results by one of our providers. 


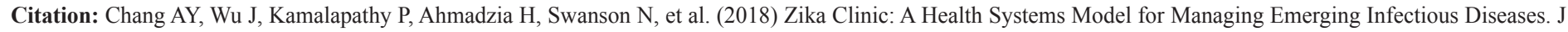
Hosp Health Care Admin: JHHA-101. DOI: 10.29011/JHHA-101. 00001

\section{Arboviral Specimen Bank}

In addition to clinical testing and counseling, Zika Clinic provides referrals to the Clinical Trials Unit for patients interested in participating in the George Washington University Arboviral Specimen Bank. The purpose of the Arboviral Specimen Bank is to collect blood and or other bodily fluids/tissues that may be used for arboviral research in the future. Samples collected from patients seen in Zika clinic have already proven useful in studies of the immunopathology of Zika disease. Given no positive serologic testing thus far, these samples have served as controls in comparison to positive serologic samples from international collaborators.

\section{Results}

A retrospective cross sectional analysis was done to characterize the patients seen in Zika Clinic. The George Washington University IRB (IRB\#021672) approved this review. All 70 patients seen in the Zika Clinic from October 2016 to March 2017 were included. Patients were referred to Zika Clinic by their obstetrician (54\%), their primary care provider (14\%), their fertility specialist $(16 \%)$, or their community provider (13\%) (Figure 2$)$. The clinic patients were predominantly females $(64 \%)$ and of those, $73 \%$ were pregnant (Table 1). All of the patients attending clinic were interested in Zika diagnostic testing and 90\% came for reproductive concerns. 17 patients reported interest in paying out of pocket for diagnostic laboratory fees if they were not eligible for free $\mathrm{DOH}$ testing and of those twelve patients did pay out of pocket for testing expenses.

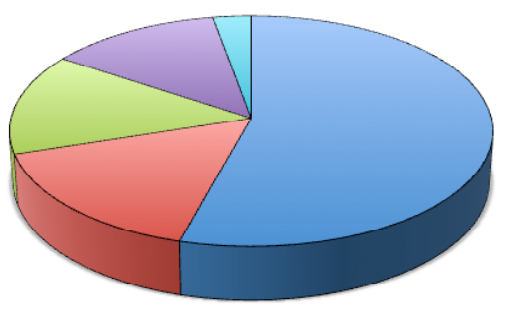

$\square$ Referred by Obstetrics, $38 / 70(54 \%)$

$\square$ Referred by Fertility Specialist, 11/70 (16\%)

$\square$ Referred by Primary Care Provider, 10/70 (14\%)

$\square$ Referred by Community, $9 / 70(13 \%)$

$\square$ Referral Unknown, 2/70 (3\%)

Figure 2: Origin of Zika Clinic referral.

\begin{tabular}{|c|c|}
\hline Characteristic & Value n (\%) \\
\hline Female Gender & $45 / 70(64 \%)$ \\
\hline Pregnant females & $33 / 45(73 \%)$ \\
\hline Interested in testing & $70 / 70(100 \%)$ \\
\hline Came for reproductive concerns & $63 / 70(90 \%)$ \\
\hline
\end{tabular}

\begin{tabular}{|c|c|}
\hline Interested in paying out of pocket & $17 / 24(71 \%)$ \\
\hline Paid out of pocket & $12 / 17(71 \%)$ \\
\hline
\end{tabular}

Table 1: Characteristics of the patients seen in Zika Clinic.

The majority of patients seen in Zika Clinic received Zika diagnostic testing (86\%) (Table 2). $63 \%$ of the patients had PCR and IgM testing while $86 \%$ of the patients had only IgM testing. All of the PCR and IgM test results have been negative to date. $16 \%$ of the total PCR testing and $24 \%$ of the total IgM testing results were pending at the time of analysis. $20 \%$ of the patients elected to send their samples to LabCorp with an average of $5 \pm 8$ days waiting time for results. $80 \%$ of the patients sent their samples to the DOH PHL with an average of $33 \pm 28$ days waiting time for results.

\begin{tabular}{|c|c|}
\hline Characteristic & Value \\
\hline Received Zika Diagnostic Testing & $60 / 70(86 \%)$ \\
\hline PCR testing, $\mathrm{n}(\%)$ & $44 / 70(63 \%)$ \\
\hline IgM testing, n (\%) & $60 / 70(86 \%) 10$ \\
\hline Both PCR and IgM testing, $\mathrm{n}(\%)$ & $44 / 70(63 \%)$ \\
\hline Positive PCR results, n (\%) & $0 / 44(0 \%)$ \\
\hline PCR results not completed date, $\mathrm{n}(\%)$ & $7 / 44(16 \%)$ \\
\hline Positive IgM results, n (\%) & $0 / 59(0 \%)$ \\
\hline IgM results not completed to date, $\mathrm{n}(\%)$ & $14 / 59(24 \%)$ \\
\hline Used LabCorp, n (\%) & $12 / 60(20 \%)$ \\
\hline $\begin{array}{l}\text { Average days to get results back from } \\
\text { LabCorp, mean } \pm \mathrm{sd}\end{array}$ & $5.16 \pm 8.28$ \\
\hline Used DOH, n (\%) & $48 / 60(80 \%)$ \\
\hline $\begin{array}{l}\text { Average days to get results back from } \mathrm{DOH} \text {, } \\
\text { mean } \pm \mathrm{sd}\end{array}$ & $33.23 \pm 27.62$ \\
\hline
\end{tabular}

Table 2: Zika testing characteristics of patients seen in Zika Clinic.

\section{Discussion}

The rationale for creating the clinic was to facilitate appropriate Zika counseling and testing in a multidisciplinary practice. There was clearly an increasing demand from patients who were requesting testing from their obstetrics/gynecologist and primary care providers as well as other specialists in our group. Given the rapidly changing environment for Zika testing it was challenging for individual providers to stay current on the recommendations for testing. The DC DOH had specific criteria for testing and those criteria were frequently changing reflecting changing recommendations from the World Health Organization and Center for Disease Control. 5 In the last year, the DC DOH issued 10 different health notices related to Zika with changing 
travel, testing, and management guidelines. There were multiple case reporting and laboratory forms making the reporting complex and time consuming. For those that willing to pay out of pocket for testing, the available tests are costly and should only be done within specific time frames to be accurate. Designing a specialized Zika Clinic with internal experts familiar with the DC DOH guidelines and evidence based guidelines for testing of Zika met both the patients' and providers' needs. Additionally, the clinic providers created a registry of patients so that when the DC DOH issued an alert that over 400 samples were incorrectly analyzed [6]. we could respond quickly and contact patients from our clinic to provide counseling regarding the meaning of this error. With summer's warmer weather and more travel to potential Zika areas, Zika screening needs could be an increasing problem for community/ providers to handle if they do not have the infrastructure in place.

\section{Conclusion}

The model for the development of a specialized outpatient clinic for an emerging infectious disease proved to be an efficient and scalable method for a healthcare system to address the provider and patient care needs during an evolving epidemic with up to date evidence based recommendations and compliance with changing department of health recommendations. This model may be useful in for health system management of future emerging infectious diseases.

\section{Prior presentations: none}

Conflict of Interest: All authors report no conflicts of interest.

Data availability: The datasets generated during and/or analyzed during the current study are available by emailing the corresponding author.

\section{Acknowledgements}

Contributors: The Zika Clinic was made possible by the support of the following individuals employed by the George Washington University and the Medical Faculty Associates: Alan Wasserman, Charles Macri, Donna Embersit, Aimee Desrosiers, Nicole Swanson, Chelsea Ware, Sirlekar Bullocks, Alison Donovan, Elizabeth Kirby, and Joanna Milder. None of the acknowledged contributors received compensation or have a financial conflict of interest.

Funders: This publication was supported by Award Numbers UL1TR001876 and KL2TR001877 from the NIH National Center for Advancing Translational Sciences. Its contents are solely the responsibility of the authors and do not necessary represent the official views of the National Center for Advancing Translational Science or the National Institute of Health.

\section{References}

1. Gulland A (2016) Zika virus is a global public health emergency, declares WHO. BMJ 352: 657.

2. Traynor K (2016) As Zika cases mount, patients seek advice from healthcare teams. Am J Health Syst Pharm 73: 1200-1204.

3. Samarasekera U, Triunfol M (2016) Concern over zika virus grips the world. The Lancet 387: 521.

4. Kindhauser MK, Allen T, Frank V, Santhana RS, Dye C (2016) Zika: The origin and spread of a mosquito-borne virus. Bull World Health Organ 94: 675-686C

5. Chang C, Ortiz K, Ansari A, Gershwin ME (2016) The Zika outbreak of the $21^{\text {st }}$ century. J Autoimmun. 68: 1-13.

6. Sun L (2017) DC lab botched zika tests involving pregnant women. The Washington Post: D.C. Politics. 